\title{
Resonant Toroidal Alfvén Eigenmodes (RTAEs) in Neutral Beam Heated Reverse Magnetic Shear Plasmas on TFTR*
}

\author{
R. Nazikian, N.N. Gorelenkov, R.V. Budny, C.Z. Cheng, G-Y. Fu \\ Princeton Plasma Physics Laboratory, P.O. Box 451, Princeton NJ 08543-0451
}

\section{Introduction}

Resonant Toroidal Alfvén Eigenmodes (RTAEs) [1,2] excited by neutral beam ions are observed in the region of the internal transport barrier in enhanced reverse shear (ERS) plasmas on TFTR. These modes occur in multiples of the same toroidal mode number in the range $n=2-4$ and appear as highly localized structures near the minimum in the q-profile with frequency near to that expected for TAEs. Unlike regular TAEs, these modes are observed in plasmas where the birth velocity of beam ions is well below the fundamental or sideband resonance condition. Theoretical analysis indicates that the Toroidicity induced Alfvén Eigenmode (TAE) does not exist in these discharges due to strong pressure gradients (of the thermal and fast ions) which moves the mode frequency down into the lower Alfvén continuum. However a new non-perturbative analysis (where the energetic particles are allowed to modify
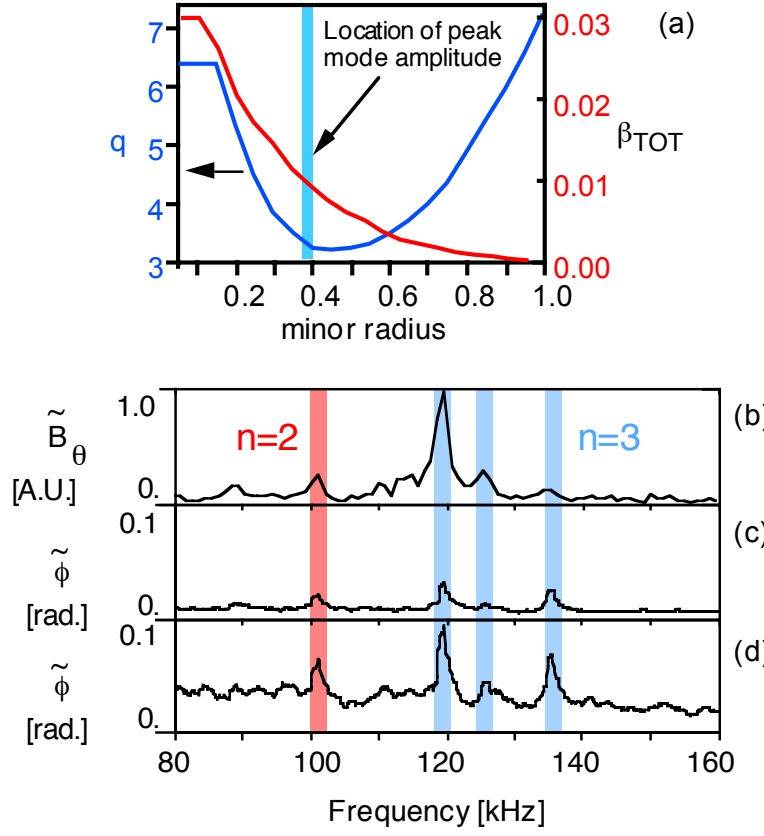

Fig. 1. Profiles of safety factor and beta (a), magnetic spectrum at plasma edge (b), reflectometer spectra at $\mathrm{r} / \mathrm{a}=0.05$ (c) and $\mathrm{r} / \mathrm{a}=0.4$ (d). Peak mode amplitude observed in weak magnetic shear region. Plasma parameters: $\mathrm{B}_{\mathrm{T}}=4.6 \mathrm{~T}, \mathrm{I}_{\mathrm{p}}=1.6 \mathrm{MA}, \mathrm{R}=260 \mathrm{~cm}, \mathrm{n}_{\mathrm{e}}(0)=7 . \times 10^{13}$ $\mathrm{cm}^{-3}, \mathrm{~T}_{\mathrm{e}}(0)=6 \mathrm{keV}, \mathrm{T}_{\mathrm{i}}(0)=21 \mathrm{keV}$. $(\# 105225)$ the mode frequency and mode structure) indicates that RTAEs can be driven by neutral beam ions in the weak magnetic shear region of ERS plasma, consistent with observations on TFTR. The importance of such modes is that they may affect the alpha particle heating profile or enhance the loss of energetic alpha particles in an advanced tokamak reactor where large internal pressure gradients and reverse magnetic shear operation are required to sustain large bootstrap current.

\section{Experimental Results}

Reverse magnetic shear operation has proven to be an effective means for enhancing the transient performance of large Tokamak plasmas, and provides a development path towards a steady state tokamak reactor. Reverse shear plasmas are formed inductively in TFTR by early neutral beam preheat of full size plasmas, in order to slow down current penetration. These plasmas often exhibit spontaneous transition to states of improved core confinement with the consequent build up of large thermal and fast particle pressure gradients. This enhanced performance is attributed to the suppression of microinstabilities and ideal MHD instabilities in the negative central shear region. However, the large pressure

\footnotetext{
* This work supported by DoE contract No. DE--AC02--76--CHO--3073.
} 


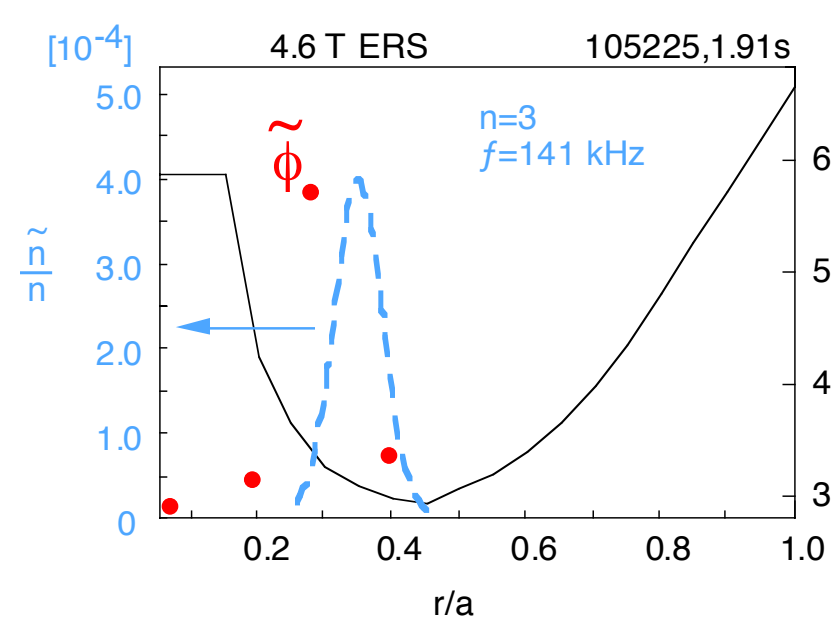

Fig. 2. Reflectometer modeling of the radial mode structure for an $n=3$ mode (dashed) together with the measured phase (circles). Modes appear in the weak magnetic shear region of the plasma.

gradient and weak magnetic shear can excite possible new instabilities in the Alfvén range of frequency. Such modes are observed in DD and DT plasmas. Unlike the usual range of TAE activity observed in weak and positive shear plasmas, the ${ }^{q}$ reverse shear modes on TFTR usually appear as closely frequency spaced modes of the same toroidal mode number with similar radial location and occur during high power neutral beam heating. [Note that in normal shear discharges with $q(0) \leq 1$, the neutral beam ions were found to be strongly stabilizing for TAEs in TFTR. (Ref. 3, 4)]

Fig. 1 shows spectra of magnetic signals along with internal reflectometer measurements for an enhanced reverse shear (ERS) plasma on TFTR. Mirnov coil spectra of external magnetic fluctuations indicate multiple (2-3) low toroidal mode number modes with weak amplitude $\left(\delta \mathrm{B} / \mathrm{B} \sim 10^{-8}\right)$ in the frequency range $100-150 \mathrm{kHz}$. The low edge amplitude may simply be an indication of the core localization of these modes. The reflectometer measurements indicate that mode activity peaks around the region of $\mathrm{q}_{\min }$. The simple expression for the TAE frequency $\mathrm{V}_{\mathrm{A}} / 4 \pi \mathrm{qR}$ is evaluated from TRANSP analysis and is in the range 120 $\mathrm{kHz}$ near $\mathrm{q}_{\min }$ - close to the observed mode frequency.

Fig. 2 shows internal measurements of the density fluctuation profile for an $n=3$ mode obtained with the core reflectometer diagnostic. The reflectometer system consists of four separate microwave frequencies which sample four radial locations on the plasma midplane. The reflectometer data is taken in $4.6 \mathrm{~T}$ discharges where the measurements range from the plasma

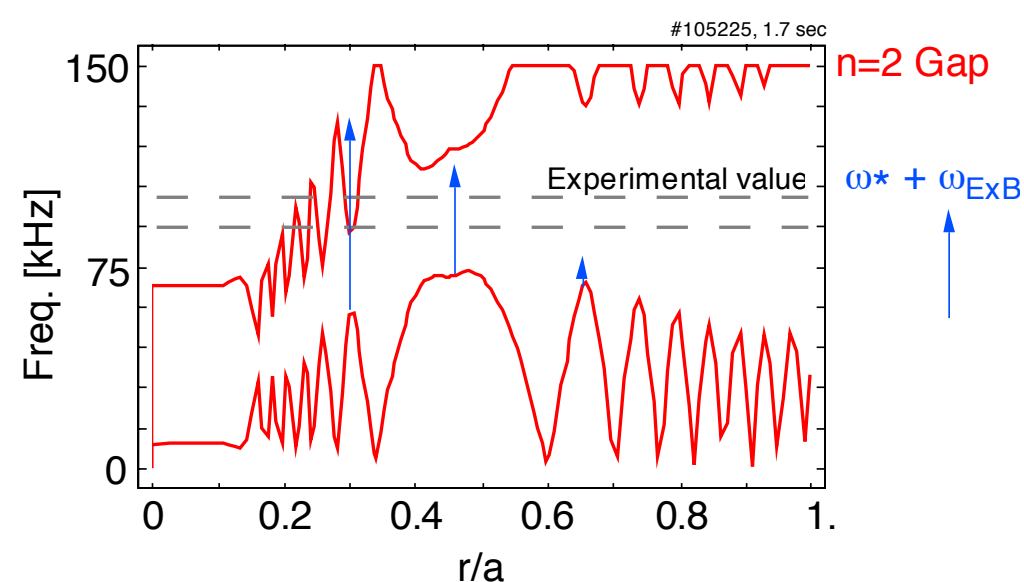

Fig. 3. Gap structure for the $n=2$ shear Alfvén continuum without correction for $\omega^{*}$ and ExB rotation. The vertical arrows indicate the magnitude of the ExB and $\omega^{*}$ correction. center out to the radius of $\mathrm{qmin}$. The density fluctuation profile is inferred from the radial profile of the measured phase fluctuations, according to geometric optics. Also shown is the MSE measured q-profile at the time of observed mode activity. The solid points are the reflectometer phase measurements while the dashed curve is the inferred location and width of the mode, assuming a Gaussian profile. The calculated radial position of the mode appears to be just inside of the radius of $\mathrm{qmin}$ at $\mathrm{r} / \mathrm{a} \approx 0.35$. Other 
data exist in which the peak of the mode is located at a radius larger than the outer most reflectometer channel, but the data is generally consistent with these modes being localized to the central weak shear region of the plasma. The internal reflectometer measurements of the mode location, external magnetic measurements of the toroidal mode number and MSE measurements of the q-profile suggest that these modes occur near the frequency expected to TAEs.

Also, the toroidal gap structure for $n=2$ TAEs is shown in Fig. 3. Unlike supershot plasmas, the high central safety factor in these regimes produces strong diamagnetic frequency shifts in the Alfvén continuum. The solid lines in the figure indicate the Toroidicity induced gap in the Alfvén continuum without $\mathrm{E}_{\mathrm{r}}$ or $\omega^{*}$ corrections to the mode frequency. The diamagnetic shift of the mode frequency is expected to lead to higher frequencies for higher toroidal mode numbers, as is observed in experiment. The magnitude of the frequency shift is indicated by the vertical arrows placed at three radial locations. It is generally found that the TAE gap reverses direction in the barrier region when the diamagnetic frequency correction is included, counteracting the effect of increasing safety factor. Similar modes are observed for a range of toroidal fields $\left(\mathrm{B}_{\mathrm{T}} \sim 2.1-4.8 \mathrm{~T}\right)$ with a frequency near the estimated $\mathrm{TAE}$ frequency evaluated at $\mathrm{q}_{\text {min. }}$. Calculations using the NOVA-K code indicate no TAE modes in the Alfvén continuum gap for the discharge in Fig. 3. This situation is attributed to the large plasma pressure $(\alpha>1)$ of thermal and beam ions at high safety factor $\left[\alpha \sim q^{2} \operatorname{grad}(\beta)\right]$. The current analysis suggests that other effects, most notably the non-perturbative contribution of the background plasma and energetic particles (not currently included in the NOVA-K analysis), are likely to play an important role in these instabilities.

HINST Analysis

A recently developed high-n stability code HINST [2] has been used to find the

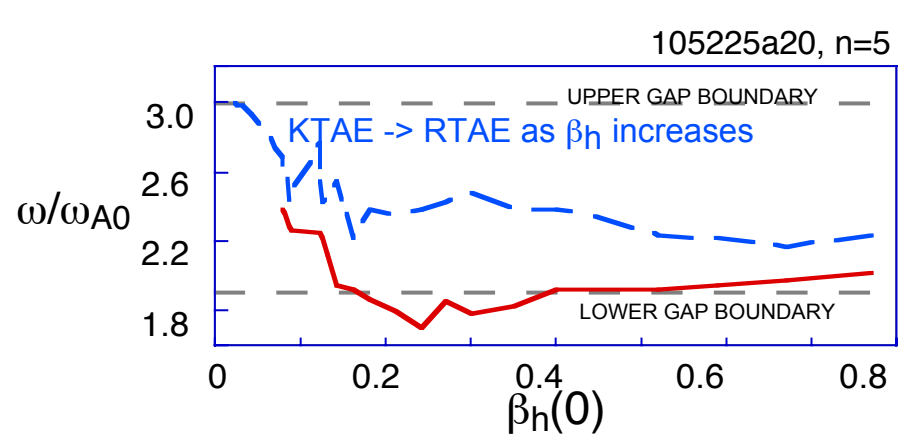
candidate modes in the Alfvén range of frequencies to explain the (a) measurements on TFTR. HINST is able to model nonperturbatively Alfvén modes in both $1 \mathrm{D}$ and 2D. It utilizes a 2D Fourier-Ballooning formalism with direct calculation of the global mode frequency and mode structure. The effect of $\omega^{*}$ is included and is shown to be important in the

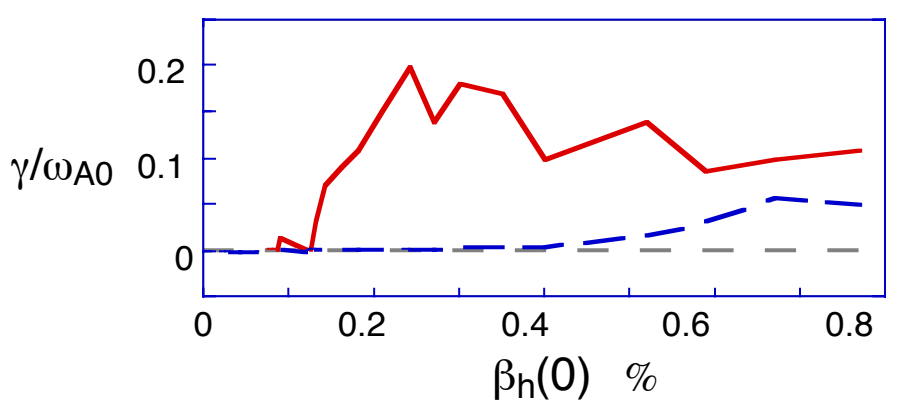

Fig. 4. Real frequency (a) and growth rate (b) calculated vs hot particle beta, obtained using the HINST high-n code. The most unstable modes are RTAEs which move to the bottom of the gap with increasing fast particle drive. (b) interpretation of the experimental data. TRANSP analysis is used to generate equilibrium profiles and fast particle distributions for input to the HINST code.

For the TRANSP analysis of shot 105225, a branch of the solution of TAE like modes is observed in the calculations near the location of the Internal Transport Barrier (ITB). The mode is driven by the injected beam ions with a slowing 
down distribution function at the cutoff energy equal to the injection energy $\approx 100-110 \mathrm{keV}$. As expected from Fig. 3, the structure of the gap indicates a strong $\omega^{*}$ effect near the center of the plasma, which is large due to the strong pressure gradient near the ITB. A sensitivity scan performed by introducing small variations to the q-profile in the HINST analysis has confirmed that these modes peak near the minimum in the q-profile. Fig. 4 shows the HINST analysis for this discharge evaluated at $\mathrm{r} / \mathrm{a}=0.5$, which corresponds to the position of the maximum linear growth rate. We identify these mode as RTAE or EPM. Such modes can not exist without fast particles. Figure 4 plots the real and imaginary mode frequency as a function of the beam ion beta, keeping the total plasma beta fixed. Two modes are identified with frequencies which drop to the bottom of the gap as the hot particle beta is increased to the value determined by TRANSP for this discharge. With no beam ion beta, there is no solution in the toroidicity induced gap, consistent with NOVA-K analysis for this discharge. However, there is an RTAE mode (according to the HINST analysis) which starts at the upper edge of the gap. As the beam beta is increased the linear growth rate of the RTAE solution becomes positive, and the mode frequency decreases rapidly towards the bottom of the gap. At finite beam beta, a new mode appears in the gap which again does not exist in the absence of fast particle drive. Together these modes move towards the bottom of the gap with a frequency separation of the order observed in experiment for two modes of the same toroidal mode number.

\section{Discussion}

An essential issue is how we can reconcile the results of the HINST analysis with our conventional understanding of Alfvén eigenmodes and their excitation. A second issue is the likely importance of such non-perturbative modes in future high beta devices.

The excitation of the RTAEs is predicted even though the usual resonance condition is not satisfied $\left(\mathrm{v}_{\mathrm{b}}<\mathrm{v}_{\mathrm{A}}\right.$ or $\left.\mathrm{v}_{\mathrm{A}} / 3\right)$. In TAE theory the mode is located where $\mathrm{m}$ and $\mathrm{m}+1$ harmonics are coupled at the $\mathrm{q}=(\mathrm{m}+1 / 2) / \mathrm{n}$ surface. With finite plasma pressure, more harmonics are activated with an amplitude which decreases roughly as $\alpha^{1}$, where $\alpha$ is the standard ballooning parameter. In case of ITB plasmas we have $\alpha \sim 1$, which results in the presence of the neighboring harmonics. For passing particles the resonant condition becomes $v_{b}=v_{A} /(1+/-21) \sqrt{ }(1-\omega * / \omega)$. One can see that increasing the 1 number and increasing $\omega^{*}$ can achieve higher order resonance, which will be weakened by the smaller amplitude of the corresponding harmonic.

In a future DT reactor based on the reverse or weak central shear tokamak concept,

there will be a large population of alpha particles capable of interacting with such RTAE modes through the fundamental or first sideband resonance. Under such conditions barrier modes may exhibit strong linear drive and large non-linear mode saturation and particle redistribution. The effect of a "sea" of high-n modes near $\mathrm{q}_{\text {min }}$ may lead to enhanced losses of fast particles, or even to a significant reduction in the central alpha heating efficiency. The further investigation of such non-perturbative instabilities needs to be carried out on present devices with the use of significant populations of passing particles. Analysis indicates that the passing particle drive is dominant for alpha driven instabilities and an important objective for future experiments is to produce a significant population of energetic passing particles in advanced tokamak regimes.

\section{References}

[1] C.Z. Cheng, N.N. Gorelenkov, C.T. Hsu, NF 351639 (1995).

[2] N. N. Gorelenkov, C. Z. Cheng, and W. Tang, Phys. Plasmas 6, 629 (1999).

[3] R. Nazikian et al., Phys. Rev. Lett., 78, 2976 (1997).

[4] G-Y. Fu, Phys. Rev. Lett., 75, 2336 (1995). 